\title{
Kissing Molars: A Report of Three Cases and Literature Review
}

\section{Luis Miguel Gonzalez-Perez*, Pedro Infante-Cossio, Marta Sanchez-Sanchez, Carlos Valdivieso-del-Pueblo and Marta Robles-Garcia}

\author{
Department of Oral and Maxillofacial Surgery, Virgen del Rocio University Hospital, Spain
}

*Corresponding author: Dr. Luis Miguel Gonzalez-Perez, Department of Oral and Maxillofacial Surgery "Virgen del Rocio" University Hospital, Av. Manuel Siurot s/n. 41013, Seville, Spain, Tel: +34 955 012608, Fax: +34 955 012576, E-mail: lumigon@telefonica.net/ovmase@gmail.com

\begin{abstract}
Objectives: The term "kissing molars" was first described by Van Hoof in 1973, in reference to teeth which have occlusal surfaces contacting each other in a single follicular space and roots pointing in opposite directions. In this study we evaluated the etiopathogenesis, clinical features, and diagnostic and therapeutic options for the management of kissing molars in the mandible.

Material and methods: MEDLINE search of articles published on the topic of "kissing molars" in the mandible through to the year 2015. Nineteen clinical cases from the literature were evaluated together with three new clinical cases provided by the authors, representing a sample of twenty-two patients.

Results: We found a mean age at diagnosis of 29.1 years. Six patients had bilateral kissing molars, while kissing molars were located unilaterally in sixteen patients: on the right side in four patients, on the left side in three patients, and the affected side is not mentioned in the remaining nine unilateral cases. The second and third molars were affected in seventeen patients, while in four patients the third and fourth molars were involved, and in one case the first and second molar were affected. Five cases were asymptomatic. Treatment included conservative management in two cases and surgical removal in seventeen patients. In the three remaining patients no data concerning their management were provided.

Conclusions: While the etiopathogenesis of kissing molars is yet to be elucidated, it seems that developmental disorders of the jaw that cause bone resorption by expansion of the follicular cyst associated with impaction of the first, second, third or fourth molars, seems to be the most appropriate explanation for the condition Surgical management must be planned according to the location and position of the kissing molars, along with an assessment of possible morbidity associated with the surgery.
\end{abstract}

\section{Keywords}

Kissing molars, Impacted mandibular molars, Inferior alveolar nerve injury, Mandible

\section{Introduction}

The existence of two mandibular impacted molars with confronted occlusal surfaces is extremely rare. When the two mandibular molars are surrounded by a single dental follicle, an entity called "kissing molars" (KM) is formed. After the first description of KM by Van Hoof in 1973 [1], very few cases have been reported in the literature. Therefore, knowledge about the etiopathogenesis, clinical features, and diagnostic and therapeutic options for KM is limited. The present study is based on a literature review of cases of mandibular KM reported through to 2015 , together with the presentation of 3 new clinical cases taken from the personal experience of the authors.

\section{Material and Methods}

A literature search of Medline/PubMed (http://www.ncbi.nlm. nih.gov/PubMed) that included articles up to June 2015 was conducted using the keyword "kissing molars". Inclusion criteria were: Englishlanguage articles generated by the Medline database containing at least one case of KM located in the mandible that detailed clinical, diagnostic or therapeutic criteria. The search revealed eleven papers that met these criteria [1-11]. Three additional cases are provided by the authors.

\section{Results}

This study included twenty-two patients with KM in the mandible, with nineteen clinical cases taken from the literature [1-11], and three new clinical cases arising from the personal experience of the authors (Table 1).

The first new clinical case that we report here involved a 29-yearold-woman with a 3-month history of swelling over the lower right side of the face. Her medical history was unremarkable, except for the diagnosis of perinatal cerebral anoxia, which left her mentally handicapped. She had undergone extraction of multiple carious teeth. On examination, there was localized pain in the right edentulous molar area. There was no evidence of palpable cervical lymphadenopathy. The remainder of the head and neck examination was normal. A panoramic radiography and a computed tomography (CT) scan were performed in which the fully impacted mandibular right third molar could be observed with its occlusal surface confronted to the right second molar's homonymous surface with destructive caries, in a distoangular position. The presence of one follicular space embracing both teeth, and with their roots in opposite directions, could also be observed (Figure 1A). The CT examination confirmed the presence of a single follicular space that included both teeth (Figures 1B,1C). Surgery was performed under endotracheal general anesthesia. A

\section{CIinMed International Library}

Citation: Gonzalez-Perez LM, Infante-Cossio P, Sanchez-Sanchez M, Valdiviesodel-Pueblo C, Robles-Garcia M (2015) Kissing Molars: A Report of Three Cases and Literature Review. Int J Oral Dent Health 1:012

Received: July 17, 2015: Accepted: August 04, 2015: Published: August 07, 2015 Copyright: (c) 2015 Gonzalez-Perez LM. This is an open-access article distributed under the terms of the Creative Commons Attribution License, which permits unrestricted use, distribution, and reproduction in any medium, provided the original author and source are credited. 
Table 1: Patients data in previous reports in the literature

\begin{tabular}{|c|c|c|c|c|c|c|}
\hline Authors/Year & Age & Gender & $\begin{array}{l}\text { Molar } \\
\text { impaction }\end{array}$ & Symptoms and associated signs & Treatment & Postoperative complications \\
\hline Van Hoof RF [1] & 31 & M & $2^{\circ} \& 3^{\circ}$ Bilateral & Not available & Not available & Not available \\
\hline Robinson JA et al. [2] & 25 & M & $2^{\circ} \& 3^{\circ}$ Bilateral & Not available & Not available & Not available \\
\hline Mclntyre G [3] & 19 & $\mathrm{~F}$ & $2^{\circ} \& 3^{\circ}$ Left & Pericoronitis & Extraction under GA & Trismus, dry socket, bone sequestrum \\
\hline Manani A [4] & 38 & M & $2^{\circ} \& 3^{\circ}$ Left & Not available & Conservative & Not available \\
\hline Bakaeen G, Baqain ZH [5] & 23 & M & $3^{\circ} \& 4^{\circ}$ Bilateral & Pain & Extraction under GA & Not available \\
\hline Krishnan B [6] & 36 & $\mathrm{~F}$ & $2^{\circ} \& 3^{\circ}$ Left & $\begin{array}{l}\text { Intra/ extraoral swelling } \\
\text { Dentigerous cyst }\end{array}$ & Extraction under LRA & Uneventful \\
\hline Boffano P., Gallesio C [7] & 42 & M & $3^{\circ} \& 4^{\circ}$ Right & Not available & Extraction under LRA & Uneventful \\
\hline Gulses A et al. [8] & 26 & $\mathrm{~F}$ & $2^{\circ} \& 3^{\circ}$ & Uneventful & Extraction & Uneventful \\
\hline Gulses A et al. [8] & 32 & $\mathrm{~F}$ & $2^{\circ} \& 3^{\circ}$ & Dentigerous cyst & Extraction & Uneventful \\
\hline Gulses A et al. [8] & 44 & M & $3^{\circ} \& 4^{a}$ & Uneventful & Extraction & Uneventful \\
\hline Gulses A et al. [8] & 23 & M & $2^{\circ} \& 3^{\circ}$ & Follicle granulomatous changes & Extraction & Paraesthesia IDN (4 months) \\
\hline Gulses A et al. [8] & 16 & $M$ & $1^{\circ} \& 2^{\circ}$ & Dentigerous cyst & Extraction & Paraesthesia IDN (6 months) \\
\hline Gulses A et al. [8] & 37 & $\mathrm{~F}$ & $2^{\circ} \& 3^{\circ}$ & Uneventful & Extraction & Uneventful \\
\hline Gulses A et al. [8] & 22 & $\mathrm{~F}$ & $2^{\circ} \& 3^{\circ}$ & Follicle granulomatous changes & Extraction & Uneventful \\
\hline Gulses A et al. [8] & 27 & M & $3^{\circ} \& 4^{\circ}$ & Dentigerous cyst & Extraction & Paraesthesia IDN (3 months) \\
\hline Gulses A et al. [8] & 20 & $\mathrm{~F}$ & $2^{\circ} \& 3^{\circ}$ & Uneventful & Extraction & Uneventful \\
\hline Sa Fortes RZ [9] & 33 & M & $2^{\circ} \& 3^{\circ}$ Bilateral & Bilateral dentigerous cysts & Extraction under LRA & Uneventful \\
\hline Kiran HY [10] & 18 & $\mathrm{~F}$ & $2^{\circ} \& 3^{\circ}$ Bilateral & Face swelling & Extraction under GA & Uneventful \\
\hline Anish N [11] & 35 & M & $2^{\circ} \& 3^{\circ}$ Bilateral & Uneventful & Not available & Not available \\
\hline $\begin{array}{l}\text { Case } 1 \text { provided by the } \\
\text { authors }\end{array}$ & 29 & $\mathrm{~F}$ & $2^{\circ} \& 3^{\circ}$ Right & Extraoral swelling & Extraction under GA & Uneventful \\
\hline $\begin{array}{l}\text { Case } 2 \text { provided by the } \\
\text { authors }\end{array}$ & 35 & M & $2^{\circ} \& 3^{\circ}$ Right & $\begin{array}{l}\text { Pain in the TMJ } \\
\text { Dentigerous cyst }\end{array}$ & Extraction under LRA & Uneventful \\
\hline $\begin{array}{l}\text { Case } 3 \text { provided by the } \\
\text { authors }\end{array}$ & 29 & $\mathrm{~F}$ & $2^{\circ} \& 3^{\circ}$ Right & Temporo-mandibular myofascial pain & Conservative follow-up & Not available \\
\hline
\end{tabular}

M: Male, F: Female, GA: General Anesthesia, LRA: Locoregional Anesthesia, IDN: Inferior Dental Nerve.
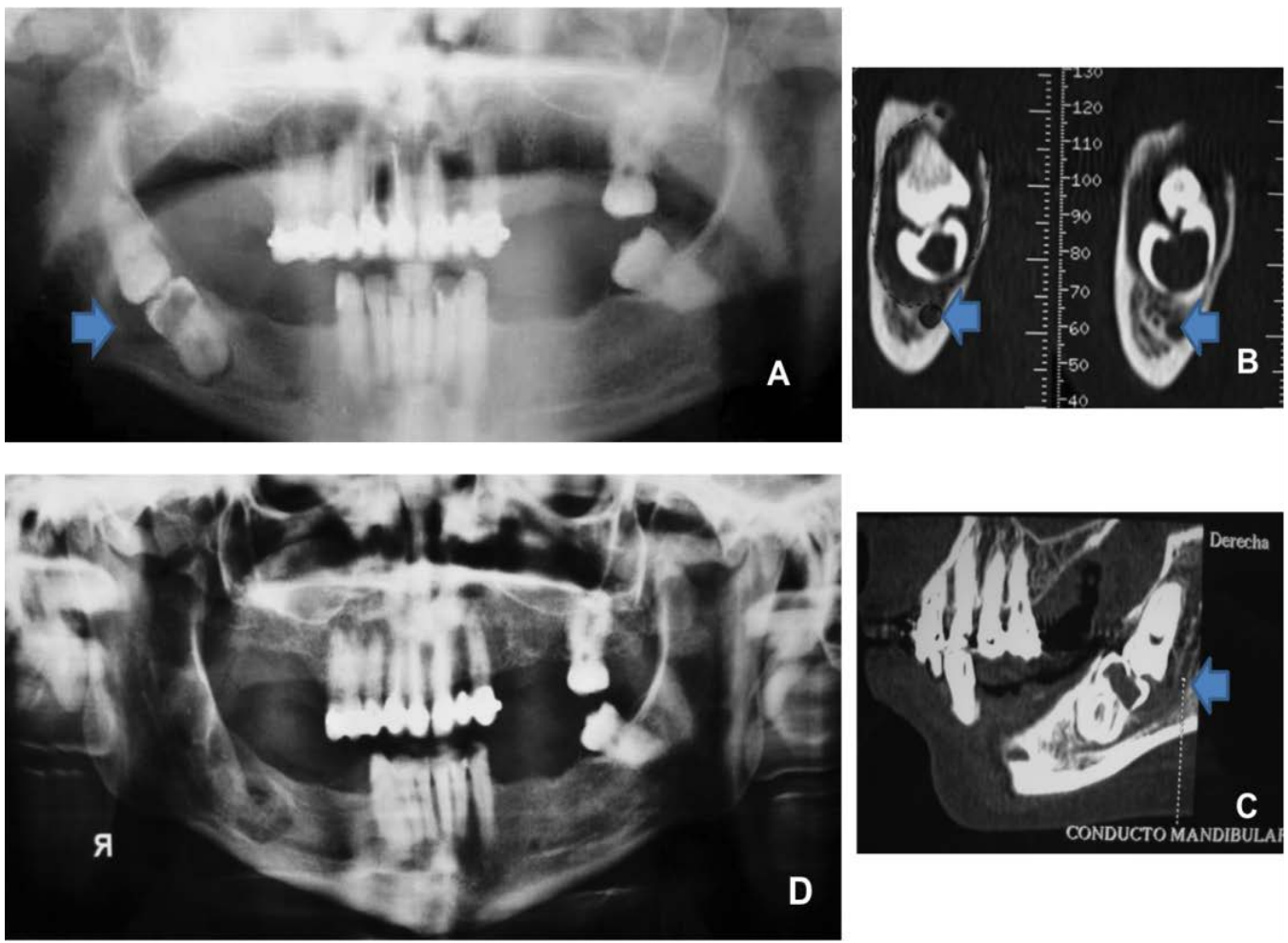

Figure 1A: Panoramic radiography with impacted mandibular second and third molars with a single follicular space.

Figure 1B (Coronal Reconstruction): CT may enable to make an accurate diagnosis of impacted molars. It is very important the role of preoperative CT imaging of the inferior alveolar nerve to determine its anatomical proximity to kissing molars (see arrow).

Figure 1C (Sagittal Reconstruction): CT images are useful for determining if there is an intimate relationship between the mandibular canal (see arrow) and the dental inclusion, as in our case.

Figure 1D: Clinical examination and imaging studies demonstrated excellent long term healing after surgery.

bayonet incision was made and a mucoperiosteal flap was elevated. Ostectomy was performed and apposition of the occlusal surfaces of these molars was seen. The necessary odontosections were made and the second molar was extracted first, followed by that of the third molar. The inferior alveolar nerve was identified intraoperatively and was left undisturbed. Associated follicular tissue was also removed 

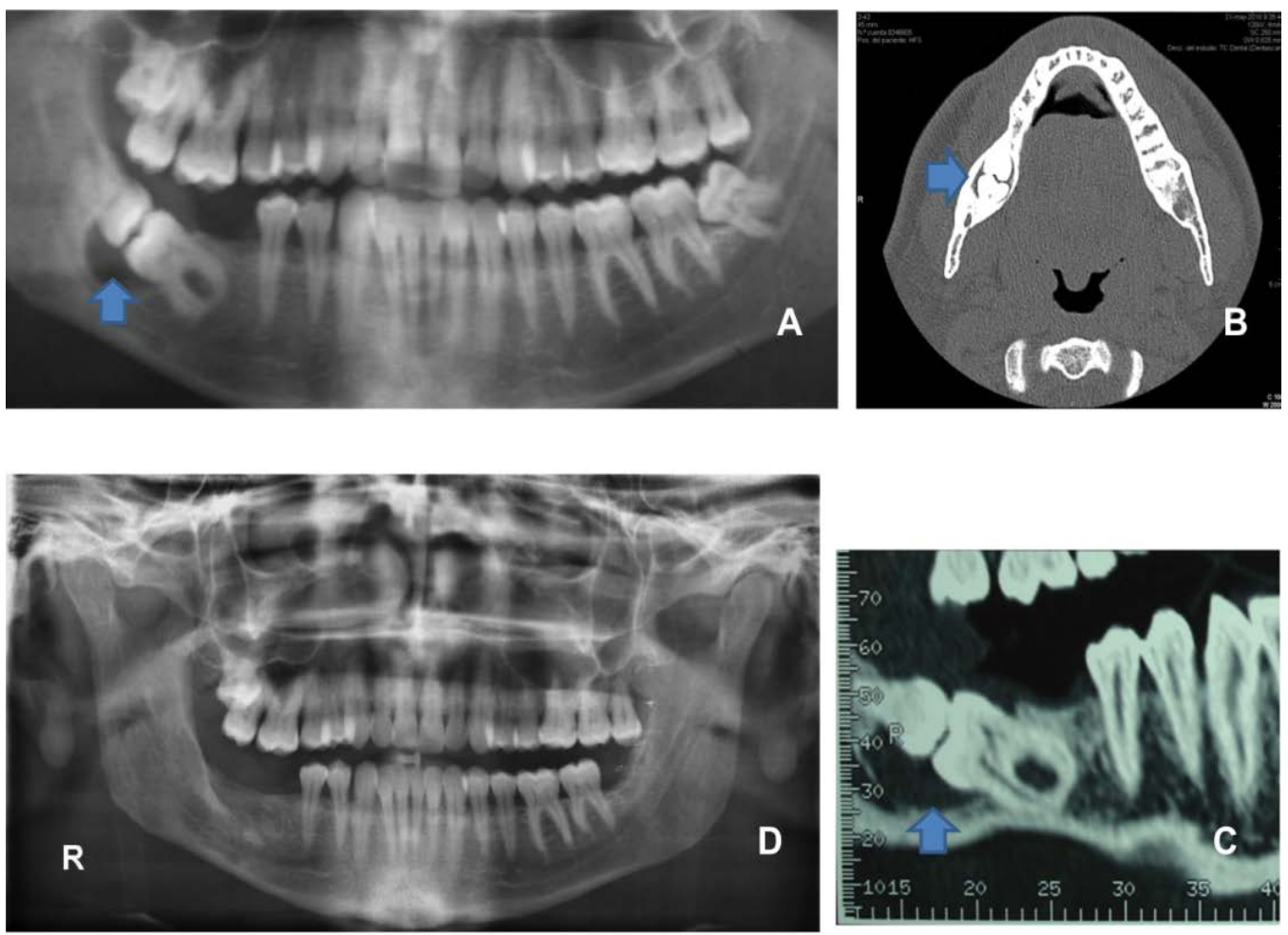

Figure 2A: Orthopantomography showing impacted right second and third mandibular molars in the position of kissing molars, where there is an intimate relationship between the mandibular canal and the cyst (see arrow).

Figure 2B (Axial Reconstruction): CT image of the patient; occlusal surfaces contacting each other in a single follicular space (see arrow).

Figure 2C (Coronal Reconstruction): The coronal sections allowed a bucco-lingual appreciation of the mandibular canal to identify cases in which a lingually placed inferior alveolar nerve is at risk during surgery, as in our case (see arrow).

Figure 2D: Clinical examination and imaging studies demonstrated excellent long term healing after surgery.

along with both molars. The residual surgical cavity was filled with a synthetic bone graft material (calcium phospho-silicate) (NovaBone') and resorbable suture was used (polyglycolic acid 4/0) for the closure of the surgical wound. No signs of infection were observed after the surgical procedure, nor were any signs of inferior alveolar nerve anesthesia or dysesthesia identified. The panoramic radiography six months later showed an excellent long term healing (Figure 1D).

The second new clinical case taken from our experience was a healthy 35 -year-old man referred with a history of pain in the temporomandibular joint in the previous three months. His medical history was unremarkable. Panoramic radiograph and CT study revealed the full impaction of the second and third mandibular right molars, with their occlusal surfaces confronted and their roots facing in opposite directions. Both impacted teeth were related to a single cyst (Figures $2 \mathrm{~A}-2 \mathrm{C}$ ). Surgical intervention was performed using locoregional anesthesia. The necessary odontosections were performed, and the second molar was extracted first followed by the third molar. An associated cyst was removed and the material was analysed. The definitive histopathological diagnosis was dentigerous cyst. The postoperative course was uneventful. No signs of inferior alveolar nerve anesthesia or dysesthesia were observed. The panoramic radiograph taken six months later demonstrated excellent long term healing (Figure 2D) and the patient was free of symptoms.

The third new clinical case that the authors report was a 29 -yearold woman referred with a history of temporomandibular myofascial pain in the previous six months. No other symptoms were reported, and TMJ examination also revealed no relevant findings. Panoramic radiography revealed horizontally impacted second and third mandibular right molars, with their occlusal surfaces confronted in same follicular space and their roots facing in opposite directions over mandibular canal (Figure 3). After considering all the factors, the authors decided follow-up of the patient without extraction.

Among the twenty-two patients reported in the present study
(Table 1), we found a higher prevalence in men, who accounted for twelve cases (55\% of the patients). The mean age was $29.1 \pm 7.9$ years, with a range from 16 to 44 years. Six patients had bilateral KM (27\%), while KM was located unilaterally in sixteen patients (73\%): on the right side in four patients and on the left side in three patients; the affected side is not mentioned in the remaining nine unilateral cases. In one of the patients with bilateral KM, the third and fourth molars were involved. The second and third molars were affected in seventeen patients (77\%), while in four patients the third and fourth molars were involved (18\%), and in one case the first and second molar were affected (5\%). A radiolucent image around the KM consisting of a single follicular space was evident in all patients by panoramic radiograph. In six patients this image turned out to be dentigerous cysts confirmed histopathologically ${ }^{5}$. The most frequent signs and symptoms were pain and swelling on the ipsilateral side of the mandible or TMJ. One patient described classical symptoms of pericoronitis. Asymptomatic KM cases were reported in five patients. Seventeen patients underwent KM extraction using either endotracheal general anaesthesia (four patients) or local anaesthesia (four patients); the employed anesthesia is not mentioned in nine cases with KM extraction. In two patients it was decided not to remove the KM and for a conservative follow-up to be conducted. In three patients no data concerning the mode of management were reported. After the surgical procedure, trismus, dry socket and bone sequestrum were observed in one patient, and signs of paraesthesia of the inferior dental nerve were observed in three patients, which resolved three to six months postoperatively. No other serious complications were reported for any of the patients after surgical intervention.

\section{Discussion}

The term KM as first described by Van Hoof in 1973 refers to teeth which have occlusal surfaces contacting each other in a single follicular space and with roots pointing in opposite directions [1]. It 


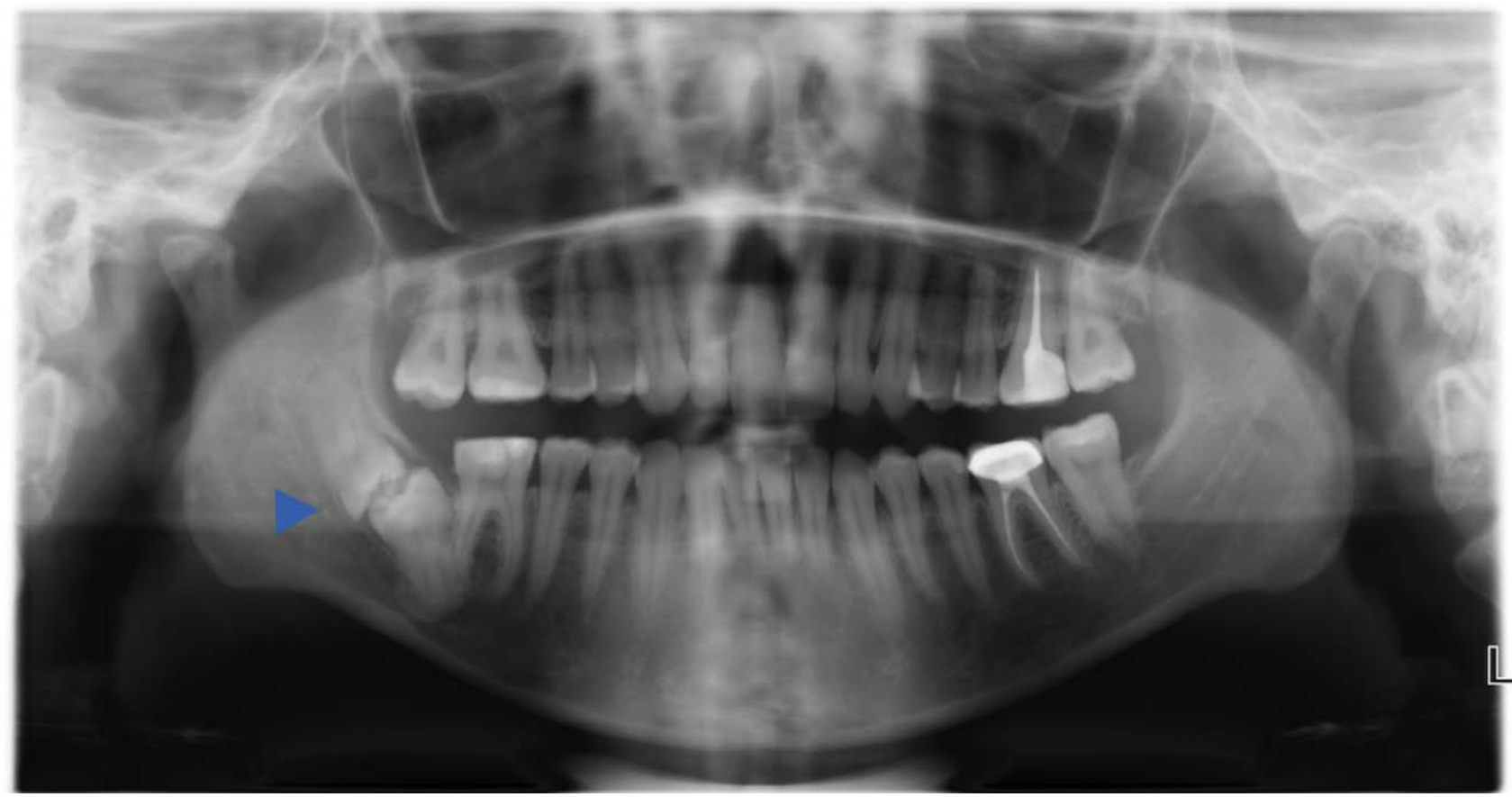

Figure 3: Kissing molars diagnosed during routine radiographic examination in asymptomatic patient showing impacted right second and third mandibular molars over mandibular canal with increased risk of nerve injury during extraction (see arrow).

is generally accepted that the phenomenon of KM involves contacting occlusal surfaces of impacted mandibular molars as evidenced by panoramic radiography $[5,7,8]$. While the etiology of KM cannot be determined definitively, it may be associated with developmental disorders of the jaw or with pathological conditions [7]. It is likely that the resorption of the bone by the expanding follicular cyst associated with the second or third molar impaction causes significant bone loss along the mesial root of the impacted third or fourth molars; this results in the molar occlusal surface coming into contact with that of the adjacent molar.

$\mathrm{KM}$ or multiple rosetting of molars has been associated with pathological conditions such as mucopolysaccharidoses and cleidocranial dysplasia [6,10.12]. Both are developmental conditions that are detected in young patients with stigmata of the syndromes, serving presence of these anomalies as an indicator to perform further investigations. Mucopolysaccharidosis type VI (MaroteauxLamy syndrome) is a disorder resulting from a genetic defect in the degradation of dermatan-sulphate, which accumulates in different tissues and results in gingival hyperplasia, malocclusion, prolonged retention of the primary dentition, and condylar defects. Cleidocranial dysplasia is an autosomal dominant inherited disease that results in a short stature, absence of clavicles, micrognathia, and delayed eruption of permanent dentition. To this extent, the possibility of metabolic disease involvement in patients can be easily ruled out by relevant clinical and histological analyses.

In our review, we identified twenty-two patients with KM in the mandible (including the new three cases provided by the authors): in one patient the first and second molar were affected, seventeen patients showed second and third molar involvement, while in four patients the third and fourth molars were implicated. Six patients presented bilateral $\mathrm{KM}$, while $\mathrm{KM}$ was located unilaterally in sixteen patients. Thus, the unilateral presentation and/or second and third molar affectation can be viewed as the most common forms of KM. However, considering the infrequent presence of first molar involvement, it would be logical to contemplate this latter phenomenon as being the most unusual form of the condition. Indeed, one could expect to find a higher frequency of second and third KM in the literature on the basis of two facts. First, one can consider that KM would remain asymptomatic for several years until the apparition of the first clinical signs and radiographic diagnosis could be established. Second, the possibility of under-reported cases in the literature should be kept in mind, given that the bilateral presentation, first and second molars or third and fourth molars affectation are the most likely to be reported because of their rarity and uniqueness.

Diagnosis is based on clinical findings together with imaging tests, mainly involving panoramic radiograph [1-11]. Imaging tests are not only used for definitive diagnosis, but also for the assessment of possible associated pathologies, for accurate localization of the $\mathrm{KM}$, and for the most appropriate treatment planning [10]. In our experience, preoperative CT imaging played a relevant role to evaluate the exact position of the inferior alveolar nerve for patients with increased risk of nerve injury during impacted KM extraction. Panoramic radiographs may be sufficient as the only form of preoperative imaging in the vast majority of cases where there is no overlap between the mandibular canal and the impacted teeth. However, in a number of cases where an intimate relationship between the mandibular canal and KM can be seen on the panoramic radiograph, CT is indicated [13]. CT is a useful diagnostic tool before surgery to minimize the possibility of nerve damage and to study the KM depth, their angulations, the shape and number of roots, their relation with the mandibular canal and ramus, and the possible perforation of cortical bone $[9,14]$.

Following diagnosis, a decision needs to be made whether to remove the KM or to refrain from treating them. It seems clear that $\mathrm{KM}$ should be treated if they cause adverse symptoms, are associated with cystic pathology, or to prevent future carious or periodontal complications. Despite the fact that no serious complications have been described in the literature after the removal of KM, it should be mentioned that complications such as possible definitive damage to the inferior alveolar nerve and a heightened risk of bone fracture could occur. KM diagnosed during routine radiographic examinations that are not associated with any disease does not require treatment, but a regular follow-up of patients must be planned in such cases [4,9]. In two of the three clinical cases presented in this article by the authors, the reasons indicating surgical treatment were to alleviate symptoms of pain and swelling.

\section{Conclusion}

In summary, taking into account the three new clinical cases described here by the authors, twenty-two documented cases have been reported in the literature, with a total of 28 mandibular KM involved. However, the possibility of under-reported cases in the 
literature should be kept in mind; this is why the true incidence of KM remains unknown and is probably underestimated. An etiopathogenic origin involving developmental disorders of the jaw that cause bone resorption due to an expanding follicular cyst with associated impaction of the second, third or fourth molars is likely to be the most probably explanation for the condition. The symptoms presented by patients include pain and swelling on the ipsilateral side of the mandible, or pericoronitis. The treatment of KM may involve a conservative approach, though in most cases surgical removal is indicated. The approach must be carefully planned according to the location and position of the KM and the possible morbidity associated with surgery. KM extraction demands an exhaustive knowledge of the regional anatomy, appropriate surgical abilities and rigorous case planning. Computed tomography can provide an additional preoperative assessment of surgical difficulty to correctly plan the extraction of the KM. Serious complications associated with the surgical removal of KM were not reported in any of the published cases.

\section{Patient Consent}

Each patient provided signed informed consent to allow the identified data collected to be used in this study.

\section{References}

1. Van Hoof RF (1973) Four kissing molars. Oral Surg Oral Med Oral Pathol 35: 284.

2. Robinson JA, Gaffney W Jr, Soni NN (1991) Bilateral 'kissing' molars. Oral Surg Oral Med Oral Pathol 72: 760 .
3. McIntyre G (1997) Kissing molars: an unexpected finding. Dent Update 24 : 373-374.

4. Manani A (1998) Kissing molars: unexpected finding. Dent Update 25: 219.

5. Bakaeen G, Baqain ZH (2005) Interesting case: kissing molars. Br J Oral Maxillofac Surg 43: 534

6. Krishnan B (2008) Kissing molars. Br Dent J 204: 281-282.

7. Boffano P, Gallesio C (2009) Kissing molars. J Craniofac Surg 20: 12691270.

8. Gulses A, Varol A, Sencimen M, Dumlu A (2012) A study of impacted love: kissing molars. Oral Health Dent Manag 11: 185-188.

9. Sa Fortes RZ, Junior VS, Modolo F, Mackowiecky E (2014) Kissing molars: Report of a case. J Oral Maxillofac Surg Med Pathol 26: 48-51.

10. Kiran HY, Bharani KS, Kamath RA, Manimangalath G, Madhushankar GS (2014) Kissing molars and hyperplastic dental follicles: report of a case and literature review. Chin J Dent Res 17: 57-63.

11. Anish N, Vivek V, Thomas S, Daniel VA, Thomas J, et al. (2015) Till Surgery do us Part: Unexpected Bilateral Kissing Molars. Clin Pract 5: 688.

12. Guven G, Cehreli ZC, Altun C, Sençimen M, Ide S, et al. (2008) Mucopolysaccharidosis type I (Hurler syndrome): oral and radiographic findings and ultrastructural/chemical features of enamel and dentin. Oral Surg Oral Med Oral Pathol Oral Radiol Endod 105: 72-78.

13. Ghaeminia H, Meijer GJ, Soehardi A, Borstlap WA, Mulder J, et al. (2009) Position of the impacted third molar in relation to the mandibular canal. Diagnostic accuracy of cone beam computed tomography compared with panoramic radiography. Int J Oral Maxillofac Surg 38: 964-971.

14. Leung YY, Cheung LK (2011) Risk factors of neurosensory deficits in lower third molar surgery: an literature review of prospective studies. Int $\mathrm{J}$ Oral Maxillofac Surg 40: 1-10. 